\title{
Polymicrobial anaerobic bacterial meningitis secondary to dermal sinus: a case report
}

\author{
Lijuan Luo $^{1 \#}$, Cuijin Wang ${ }^{2 \#}$, Nan Shen $^{1}$, Ruike Zhao $^{3}$, Yue Tao ${ }^{3}$, Xi Mo ${ }^{3}$, Qing Cao ${ }^{1}$ \\ ${ }^{1}$ Department of Infectious Diseases, Shanghai Children's Medical Center, School of Medicine, Shanghai Jiao Tong University, Shanghai, China; \\ ${ }^{2}$ Department of Neurology, Shanghai Children's Medical Center, School of Medicine, Shanghai Jiao Tong University, Shanghai, China; ${ }^{3}$ The \\ Laboratory of Pediatric Infectious Diseases, Pediatric Translational Medicine Institute, Shanghai Children's Medical Center, School of Medicine, \\ Shanghai Jiao Tong University, Shanghai, China \\ "These authors contributed equally to this work. \\ Correspondence to: Qing Cao. Department of Infectious Diseases, Shanghai Children's Medical Center, 1678 Dongfang Road, Shanghai 200127, \\ China. Email: caoqing@scmc.com.cn; Xi Mo. The Laboratory of Pediatric Infectious Diseases, Pediatric Translational Medicine Institute, Shanghai \\ Children's Medical Center, 1678 Dongfang Road, Shanghai 200127, China. Email: xi.mo@shsmu.edu.cn.
}

\begin{abstract}
Anaerobic bacterial meningitis is a rare infectious disease, and there are some special predisposing factors for it. We report a case of polymicrobial anaerobic bacterial meningitis in a nine-monthold boy who visited our hospital due to "fever with drowsiness and vomiting for 2 days". It was confirmed by the method of sanger sequencing after polymerase chain reaction (PCR) that the purulent meningitis was caused by a mixture of four anaerobic bacteria (Finegoldia magna, Campylobacter ureolyticus, Bacteroides fragilis and Porphyromonas bennonis). Even though there was no obvious structural abnormality on the skin surface, magnetic resonance imaging (MRI) examination suggested the presence of a sacrococcygeal dermal sinus. It was proven that anaerobic bacterial meningitis was secondary to retrograde infection of the dermal sinus. Finally, he was cured by a combination of anti-infection measures and surgical treatment. In conclusion, using appropriate molecular diagnostic techniques may quickly and accurately determine the pathogenic bacteria of anaerobic bacterial meningitis. When anaerobic bacterial meningitis occurs, the presence of structural abnormalities such as dermal sinus needs to be ruled out to avoid recurrence of the disease. In addition to anti-infective treatment, patients with dermal sinuses should undergo surgery as soon as possible to address abnormal structures and their root causes.
\end{abstract}

Keywords: Anaerobic bacterial meningitis; dermal sinus; molecular diagnostic techniques; case report

Submitted May 12, 2021. Accepted for publication Aug 12, 2021.

doi: $10.21037 /$ tp-21-210

View this article at: https://dx.doi.org/10.21037/tp-21-210

\section{Introduction}

Anaerobic meningitis is a very rare infectious disease. It has been reported that anaerobic meningitis accounts for only $2.4 \%$ of bacterial meningitis (1). Its clinical manifestations include fever, headache, vomiting, lethargy, irritability and convulsions. Cerebrospinal fluid (CSF) examination shows an increased white blood cell (WBC) count and protein concentration and a decreased glucose concentration. These characteristics are not specific and therefore cannot be differentiated from other forms of bacterial meningitis (2). The diagnosis of anaerobic meningitis depends on the isolation of pathogenic bacteria from CSF. At present, CSF culture is the gold standard for diagnosis, but in special cases, the positive rate of CSF culture is not high: (I) CSF

^ ORCID: 0000-0001-7740-8416. 
culture is substantially subject to the use of antibiotics before lumbar puncture, which reduces the positive rate of CSF culture from $60-90 \%$ to $25.6-60 \%$ (3-5); (II) anaerobic culture is not a routine process in CSF culture, so some diagnoses are missed $(1,6)$; (III) bacterial culture is time-consuming; so timely diagnosis cannot be made. This condition leads to the misdiagnosis and mistreatment of anaerobic meningitis, affects the prognosis and leads to a high fatality and disability rate. Molecular diagnostic technology provides a better way to diagnose encephalitis and meningitis. Compared with conventional bacterial culture, molecular diagnostic method has three advantages: (I) it is less affected by the use of antibiotics; (II) it is not subject to bacterial growth conditions; and (III) it is fast and provides better support for rapid diagnosis and timely treatment (7). This method shows obvious advantages, especially in complex or mixed infectious diseases (7-9). However, choosing an appropriate method among the numerous molecular diagnostic techniques is a problem for clinicians.

There are some special predisposing factors of anaerobic meningitis. Clinicians should screen for predisposing factors, including otolaryngological infection (60\%), gastrointestinal infection (10\%), premature birth (10\%), other causes (5\%; congenital dermal sinus, head and neck tumor, ventricular shunt) and unknown causes (15\%) (10). The dermal sinus is an abnormal channel between the skin surface and the central nervous system in the dorsal midline and is mostly discovered in the lumbosacral region. Through the dermal sinus, colonizing bacteria on the skin surface and even bacteria in feces can enter the central nervous system and cause infection $(11,12)$. The most common manifestation of the dermal sinus is an abnormal opening on the skin surface and pigmentation on the skin; some of the surface is covered with hair or liquid discharged from the sinus orifice, and there is a purulent secretion after infection. These patients are often asymptomatic at birth, and the ostium is relatively concealed. Moreover, some sinus orifices do not have obvious abnormal structures on the skin surface. In addition, health care doctors are not aware of such diseases, so the diagnosis of these diseases is often delayed. Ramnarayan et al. found a significant delay (mean 5 years; range, $2-18$ years) from midline lesion identification to neurosurgical intervention (13).

In this paper, we report the clinical data of a 9-month-old boy who visited our hospital due to "fever with drowsiness and vomiting for 2 days". Two CSF cultures were negative. Subsequently, polymerase chain reaction (PCR) with
Sanger sequencing aided in the diagnosis of anaerobic bacterial meningitis due to mixed bacterial infection, and spinal magnetic resonance imaging (MRI) further suggested central infection secondary to infection of the sacral dermal sinus, the root cause. In addition, surgery of the dermal sinus can promote recovery from the disease and prevent recurrence of the disease and further neurological damage. We hope that this case report will serve as a reference for the clinical diagnosis and treatment of similar cases. We present the following case in accordance with the CARE reporting checklist (available at https://dx.doi.org/10.21037/ tp-21-210).

\section{Case presentation}

A 9-month-old boy was admitted to our hospital due to "fever with drowsiness and vomiting for 2 days." At admission, physical examination showed the following observations: anal temperature $38.8^{\circ} \mathrm{C}$, heart rate $168 \mathrm{bpm}$, respiratory frequency 35 breaths per minute, blood pressure $72 / 39 \mathrm{mmHg}$, length $60 \mathrm{~cm}$, weight $8 \mathrm{~kg}$, head circumference $46 \mathrm{~cm}$, drowsiness, equally large pupils with sensitive light reflection, bulging bregma with tension, strong and rhythmic heart beats, clear breathing sounds with no rales, soft and smooth abdomen with no mass, neck resistance (+), Brudzinski's sign (+), Kernig sign (-), bilateral Babinski sign (+), knee jerk reflex (+), normal muscle tone in the limbs, no rashes, no skin ulceration, and no abnormal superficial structures. The patient had no recent cough, nasal congestion, runny nose, external ear canal discharge, vomiting, diarrhea, bloody stools or crying when urinating. The patient was born at full term via vaginal delivery, with a birth weight of $3.2 \mathrm{~kg}$. He had no history of past illness, surgery or trauma. After birth, he received vaccinations to prevent hepatitis $\mathrm{B}$, tuberculosis, diphtheria pertussis tetanus (DPT), polio, meningitis and measles. Laboratory tests showed a WBC count of $20.5 \times 10^{9} / \mathrm{L}$, lymphocytes $13.3 \%$, neutrophils $78.2 \%$, C-reactive protein (CRP) $88 \mathrm{mg} / \mathrm{L}$, procalcitonin (PCT) $3.7 \mathrm{ng} / \mathrm{mL}$, erythrocyte sedimentation rate (ESR) $35 \mathrm{~mm} /$ h. CSF tests showed WBC $16,640 \times 10^{6} / \mathrm{L}$, multinuclear cells $65 \%$, mononuclear cells $35 \%$, Pandy's test +++ , glucose $<1.1 \mathrm{mmol} / \mathrm{L}$ and protein $2,282 \mathrm{mg} / \mathrm{L}$. CSF and blood cultures were performed; CSF smear was negative. The patient was diagnosed with purulent meningitis and received empirical anti-infective therapy with vancomycin (VA) and ceftriaxone (CRO). After 5 days of treatment, the patient's mental state improved, vomiting resolved, and his 


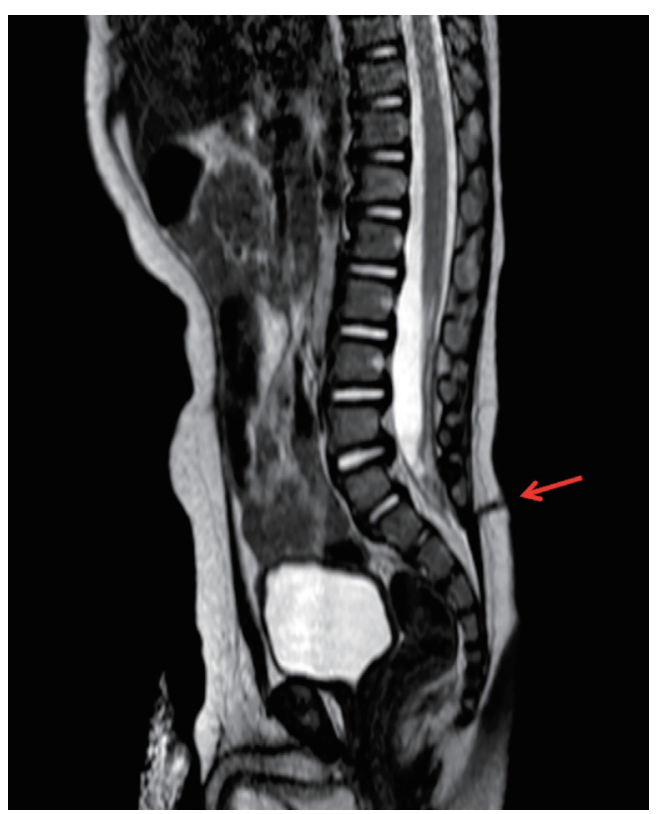

Figure 1 Spinal MRI of the patient. The red arrow shows that in the fifth lumbar segment, skin surface communicates with spinal cord cavity through the dermal sinus. MRI, magnetic resonance imaging.

temperature returned to normal. Laboratory tests showed WBC $12.2 \times 10^{9} / \mathrm{L}$, lymphocytes $38.1 \%$, neutrophils $55.2 \%$ and CRP $36 \mathrm{mg} / \mathrm{L}$. CSF tests showed WBC $6 \times 10^{6} / \mathrm{L}$, Pandy's test (-), glucose $2.0 \mathrm{mmol} / \mathrm{L}$ and protein $621 \mathrm{mg} / \mathrm{L}$. Initial CSF and blood cultures were negative. On day 7 , fever returned (up to $38.5^{\circ} \mathrm{C}$ ). Laboratory tests showed WBC $18.24 \times 10^{9} / \mathrm{L}$, neutrophils $43 \%$, CRP $57 \mathrm{mg} / \mathrm{L}$, PCT $2.3 \mathrm{ng} / \mathrm{mL}$ and ESR $42 \mathrm{~mm} / \mathrm{h}$. CSF tests showed WBC $115 \times 10^{6} / \mathrm{L}$, multinuclear cells $45 \%$, mononuclear cells $55 \%$, Pandy's test (+), glucose $1.9 \mathrm{mmol} / \mathrm{L}$ and protein $771 \mathrm{mg} / \mathrm{L}$. Both CSF smear and culture were negative. For further clarification, PCR and Sanger sequencing were performed with CSF samples. PCR and Sanger sequencing of the first CSF sample identified Finegoldia magna, Campylobacter ureolyticus, Bacteroides fragilis, and Porphyromonas bennonis, which were also identified with the second CSF sample after the symptoms recurred.

Immune indicators such as peripheral complete blood count, immunoglobulins, lymphocyte subset count and percentage, and complements were normal. No abnormality was found in routine stool and urine examinations; otitis media, sinusitis and pharyngeal abscess were ruled out using otoscopy, nasopharyngeal computed tomography (CT), and middle ear and mastoid CT; brain MRI showed slightly enlarged bilateral ventricles and no obvious changes in density and morphology in the parenchyma. Several physical examinations showed no rashes, skin ulceration, or abnormal superficial structures. To further rule out structural abnormalities, the patient underwent spinal MRI, which showed a dermal sinus at L5 that was connected to the spinal cavity, suggesting infection in the sacral spinal cavity (Figure 1). Finally, the patient was diagnosed with anaerobic bacterial meningitis secondary to retrograde infection of the sacral dermal sinus.

After the pathogens were identified, the patient started to receive meropenem and metronidazole (MNZ), but he still had recurrent fever and thus underwent resection of the lumbosacral dermal sinus after 4 weeks of anti-infective treatment while continuing to receive anti-infective treatment after surgery. His temperature returned to normal by day 3 after surgery, and anti-infective treatment was discontinued in a few weeks (total treatment time: 7 weeks). Inflammatory indicators and CSF index and temperature were regularly monitored during treatment (Table 1, Figure 2). The patient was doing well, with no purulent meningitis during the 3-year follow-up.

All procedures performed in studies involving human participants were in accordance with the ethical standards of the institutional and/or national research committee(s) and with the Helsinki Declaration (as revised in 2013). Written informed consent was obtained from the patient's parent or legal guardian for publication of this case report and accompanying images. A copy of the written consent is available for review by the editorial office of this journal.

\section{Discussion}

CSF culture is the gold standard for the diagnosis of bacterial meningitis. However, due to the aforementioned reasons, the efficiency of its clinical application was reduced in the diagnosis of anaerobic meningitis. A molecular diagnostic method, with the advantages of rapidity, high sensitivity and specificity, can improve the efficiency of pathogen diagnosis of central nervous system infection and open up a new method for rapid diagnosis of bacterial meningitis. The current molecular diagnosis methods for pathogen detection include PCR, gene chip and nextgeneration sequencing (NGS). PCR is one of the earliest molecular diagnostic techniques. In this case, Sanger sequencing after PCR is a universal primer PCR method for the detection of bacteria and fungi. This is an economical and fast detection method, but one disadvantage of this 
Table 1 Inflammatory indicators and CSF index during treatment

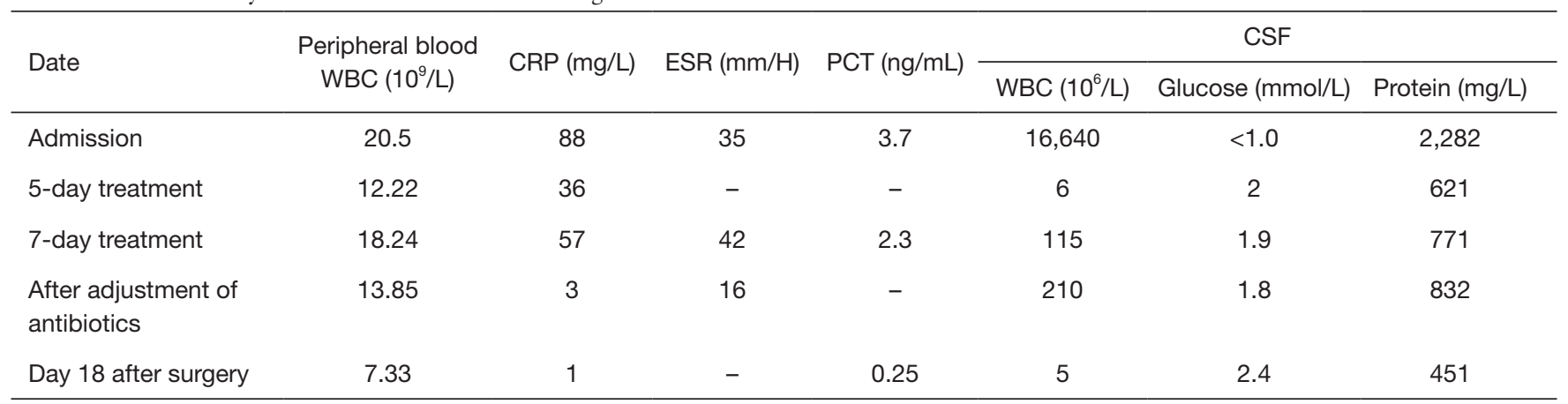

After 5 days of treatment, peripheral blood WBC, CRP and CSF indexes of the patient were improved. The indexes were repeated on the 7 th day of treatment and did not improve after adjustment of antibiotics. The indexes returned to normal 18 days after operation. CSF, cerebrospinal fluid; WBC, white blood cell; CRP, C-reactive protein; ESR, erythrocyte sedimentation rate; PCT, procalcitonin.

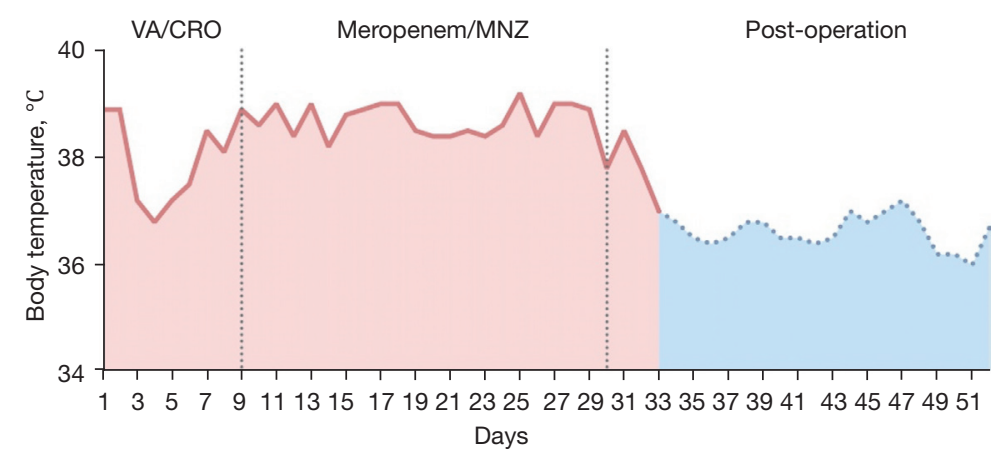

Figure 2 Temperature during treatment. On admission the patient was diagnosed with purulent meningitis and received empirical antiinfective therapy with VA and CRO. By day 9 after admission the pathogens were identified, the patient started to receive meropenem and MNZ, but he still had recurrent fever and thus underwent resection of lumbosacral dermal sinus by day 30 after admission, while continuing to receive anti-infective treatment after surgery. His temperature returned to normal by day 3 after surgery, and anti-infective treatment was discontinued in a few weeks (total treatment time: 7 weeks). VA, vancomycin; CRO, ceftriaxone; MNZ, metronidazole.

method is that it cannot detect pathogens other than bacteria and fungi. In recent years, the Filmarry meningitis/ encephalitis (ME) panel, a multiplex PCR detection technology, has been widely used. It can detect multiple pathogens in a short time (approximately 1 hour) and has high sensitivity and specificity $(14,15)$. It can detect 14 common pathogens, including bacteria, viruses and fungi, in community-acquired ME. In the diagnosis of communityacquired ME, its application value is very high. However, the relatively high detection cost, equipment requirements and limited detection range of targeted pathogens are deterrents to its use. Gene-chip technology has expanded the species of pathogens for parallel detection, with the characteristics of high flux, high parallelism and miniaturization. However, low sensitivity and high detection costs are its drawbacks. Generally, the pathogen spectrum that these detection methods can cover is gradually expanding, but these methods are always limited in the scope of targeted detection and cannot achieve full coverage of the pathogen spectrum, which will cause missed diagnoses of rare bacteria or new pathogens. NGS technology, also known as highthroughput sequencing, can sequence all the nucleic acids in the sample after removing the interference of human genome information. It overcomes the limitations of targeted diagnostic methods and has significant advantages in the detection of rare and unknown pathogens. With the long-term survival of patients with immune deficiency, immunosuppression, shunting after neurosurgical operation or implantation of other intracranial devices, the incidence of central infection in such special patients is increasing, which is usually the result of multiple or rare pathogen infection. The advantages of NGS are highlighted in this 
special type of infection $(7,16)$. However, this method has higher requirements for equipment, sites and personnel, resulting in more expensive detection costs. In addition, there is no unified standard for the definition of background bacteria, polluting bacteria, and responsible pathogens, or for interpretation of results. Further exploration is needed to improve the credibility of NGS. Therefore, molecular diagnosis technology is a powerful supplement to traditional diagnostic methods, which greatly improves the diagnostic process. However, in practical applications, we need to choose the appropriate method according to the source of infection, susceptibility factors, disease development, and local technical proficiencies. As molecular diagnostic techniques become part of routine testing, clinicians will need to use them wisely in the right clinical settings.

Delayed diagnosis of the dermal sinus often leads to serious complications such as repeated infection (including sinus infection, meningitis, spinal cord abscess, brain abscess, etc.), neurological impairment (such as lower extremity motor weakness, sensory changes, reflex changes, intestinal or bladder function changes or gait changes). A report showed that approximately $18 \%$ of patients developed infections, and approximately $30 \%$ of the cases showed neurological deficit symptoms $(13,17)$. An article summarized the condition of 28 dermal sinus patients over 30 years. Eight of 16 patients $(50 \%)$ diagnosed younger than 1 year had neurologic deficits; in contrast, 11 of 12 patients (92\%) diagnosed older than 1 year had neurologic deficits. With the delay of diagnoses, the cases of neurological impairment will be increasingly serious (18). Surgery is an important treatment for the dermal sinus and should be performed as soon as possible after diagnosis (19). Timely diagnosis and treatment of the dermal sinus play an important role in improving the prognosis and quality of life of such patients. In this case, there was no abnormal skin opening on the skin surface during the physical examination. However, due to the specificity of the patient's condition, we did not stop exploration, and MRI examination of the spinal cord was performed. Finally, through MRI examination, the existence of the sacrococcygeal dermal sinus was confirmed, and the culprit behind the infection was revealed. The patient still had recurrent fever after we adjusted antibiotics according to the pathogen and finally improved and recovered after surgical resection of the dermal sinus. Therefore, under the premise of high suspicion, doctors need to conduct a comprehensive examination to reveal the host factors behind the cases of infection. In addition to antibiotic treatment, surgery is needed to clear the focal infection and cut off the abnormal pathway to fundamentally solve the problem.

In conclusion, anaerobic bacterial meningitis may be diagnosed rapidly and accurately with appropriate molecular diagnostic techniques. Clinicians should screen for potential predisposing factors in patients with anaerobic bacterial meningitis. The dermal sinus may not present any obvious abnormal structure on the skin surface, and spinal MRI should be performed in highly suspected cases. In addition to anti-infective treatment, patients with dermal sinus and central infection should undergo surgery as soon as possible to address abnormal structures and their root causes.

\section{Acknowledgments}

We would like to thank the patient and his parents for the support and cooperation in publishing this work.

Funding: This work was financially sponsored by The Interdisciplinary Program of Shanghai Jiao Tong University (project number YG2021QN108).

\section{Footnote}

Reporting Checklist: The authors have completed the CARE reporting checklist. Available at https://dx.doi. org/10.21037/tp-21-210

Conflicts of Interest: All authors have completed the ICMJE uniform disclosure form (available at https://dx.doi. org/10.21037/tp-21-210). The authors have no conflicts of interest to declare.

Ethical Statement: The authors are accountable for all aspects of the work in ensuring that questions related to the accuracy or integrity of any part of the work are appropriately investigated and resolved. All procedures performed in studies involving human participants were in accordance with the ethical standards of the institutional and/or national research committee(s) and with the Helsinki Declaration (as revised in 2013). Written informed consent was obtained from the patient's parent or legal guardian for publication of this case report and accompanying images. A copy of the written consent is available for review by the editorial office of this journal.

Open Access Statement: This is an Open Access article distributed in accordance with the Creative Commons Attribution-NonCommercial-NoDerivs 4.0 International 
License (CC BY-NC-ND 4.0), which permits the noncommercial replication and distribution of the article with the strict proviso that no changes or edits are made and the original work is properly cited (including links to both the formal publication through the relevant DOI and the license). See: https://creativecommons.org/licenses/by-nc-nd/4.0/.

\section{References}

1. Lee JJ, Lien CY, Chien CC, et al. Anaerobic bacterial meningitis in adults. J Clin Neurosci 2018;50:45-50.

2. Brook I. Meningitis and shunt infection caused by anaerobic bacteria in children. Pediatr Neurol 2002;26:99-105.

3. van de Beek D, Cabellos C, Dzupova O, et al. ESCMID guideline: diagnosis and treatment of acute bacterial meningitis. Clin Microbiol Infect 2016;22 Suppl 3:S37-62.

4. Kanegaye JT, Soliemanzadeh P, Bradley JS. Lumbar puncture in pediatric bacterial meningitis: defining the time interval for recovery of cerebrospinal fluid pathogens after parenteral antibiotic pretreatment. Pediatrics 2001;108:1169-74

5. Wu L, Han W, Wang G, et al. Pathogenic and diagnostic analysis of bacterial meningitis children in the Midwest area. Chinese Journal of Applied Clinical Pediatrics 2018;33:778-82.

6. Pittman ME, Thomas BS, Wallace MA, et al. Routine testing for anaerobic bacteria in cerebrospinal fluid cultures improves recovery of clinically significant pathogens. J Clin Microbiol 2014;52:1824-9.

7. Guo LY, Li YJ, Liu LL, et al. Detection of pediatric bacterial meningitis pathogens from cerebrospinal fluid by next-generation sequencing technology. J Infect 2019;78:323-37.

8. Hu HL, Guo LY, Wu HL, et al. Evaluation of next-

Cite this article as: Luo L, Wang C, Shen N, Zhao R, Tao Y, Mo X, Cao Q. Polymicrobial anaerobic bacterial meningitis secondary to dermal sinus: a case report. Transl Pediatr 2021;10(11):3118-3123. doi: 10.21037/tp-21-210 generation sequencing for the pathogenic diagnosis of children brain abscesses. J Infect 2019;78:323-37.

9. Guo LY, Feng WY, Guo X, et al. The advantages of nextgeneration sequencing technology in the detection of different sources of abscess. J Infect 2019;78:75-86.

10. Gutierrez M, Emmanuel PJ. Expanding Molecular Diagnostics for Central Nervous System Infections. Adv Pediatr 2018;65:209-27.

11. Frank GR, Rubin LG. Mixed meningitis with Bacteroides ovatus caused by an occult congenital dermal sinus. Pediatr Infect Dis J 1989;8:401-3.

12. Givner LB, Baker CJ. Anaerobic meningitis associated with a dermal sinus tract. Pediatr Infect Dis 1983;2:385-7.

13. Ramnarayan R, Dominic A, Alapatt J, et al. Congenital spinal dermal sinuses: poor awareness leads to delayed treatment. Childs Nerv Syst 2006;22:1220-4.

14. O'Brien MP, Francis JR, Marr IM, et al. Impact of Cerebrospinal Fluid Multiplex Assay on Diagnosis and Outcomes of Central Nervous System Infections in Children: A Before and After Cohort Study. Pediatr Infect Dis J 2018;37:868-71.

15. Liesman RM, Strasburg AP, Heitman AK, et al. Evaluation of a Commercial Multiplex Molecular Panel for Diagnosis of Infectious Meningitis and Encephalitis. J Clin Microbiol 2018;56:e01927-17.

16. Law DA, Aronoff SC. Anaerobic meningitis in children: case report and review of the literature. Pediatr Infect Dis J 1992;11:968-71.

17. Foster MT, Moxon CA, Weir E, et al. Dermal sinus tracts. BMJ 2019;366:15202.

18. Ackerman LL, Menezes AH. Spinal congenital dermal sinuses: a 30-year experience. Pediatrics 2003;112:641-7.

19. Wang GY, Liu B, Han X, et al. Diagnosis and surgical treatment of dermal sinus tracts in children. Journal of Clinical Pediatric Surgery 2019;18:99-102. 